\title{
La définition de la communication publique : des enjeux disciplinaires aux changements de paradigmes organisationnels
}

\section{Dominique Bessières}

\section{CpenEdition}

\section{Journals}

Édition électronique

URL : http://journals.openedition.org/communicationorganisation/686

DOI : 10.4000/communicationorganisation.686

ISSN : $1775-3546$

Éditeur

Presses universitaires de Bordeaux

Édition imprimée

Date de publication : 1 juin 2009

Pagination : 14-28

ISSN : 1168-5549

Référence électronique

Dominique Bessières, « La définition de la communication publique : des enjeux disciplinaires aux changements de paradigmes organisationnels », Communication et organisation [En ligne], 35 | 2009, mis en ligne le 01 décembre 2012, consulté le 10 décembre 2020. URL : http:// journals.openedition.org/communicationorganisation/686 ; DOI : https://doi.org/10.4000/ communicationorganisation.686 
Dossier : Repenser la communication des organisations publiques

\title{
Résumé
}

Le travail social de communication institutionnelle publique est une pratique avant d'être un enjeu de définition théorique. Pourquoi cette activité organisationnelle est-elle qualifiée de façon dominante «communication publique» bien que sa définition ne soit pas totalement stabilisée? Son succès repose sur des processus d'institutionnalisation et d'intégration, une dimension gestionnaire légitimante qui la renforce. Ce champ social représente des enjeux disciplinaires à caractériser.

\section{Mots clés}

Communication publique, communication politique, politique publique, bureaucratie, new management public.

\begin{abstract}
Public communication in French context is first of all a practice. It's only on a second step, that the question of definition is appearing. The purpose of the article is to identify behind the variety of definitions of public communication, struggles of interests between academic positions. But public communication is also an action of public organizations submited at its logic and some management approach. In this context, it's interesting to underline some premises for a change of concept in public organisation that could influence practices.
\end{abstract}

\section{Key words}

Public communication, political communication, policies, bureaucracy, new management public.

Dominique Bessières est Maître de Conférences en Sciences de l'Information et de la Communication à l'Université de Reims Champagne-Ardennes. Il est responsable Master de Communication publique de l'IEP de Lille. 


\title{
La définition de la communication publique : des enjeux disciplinaires aux changements de paradigmes organisationnels
}

\author{
Dominique Bessières
}

dominique.bessieres@iep.univ-lille2.fr

On s'accorde à considérer que l'on est passé de l'information ${ }^{1}$ à la communication (Boure, 1992). Ce constat est tiré de la littérature des années 70 en comparaison de celle des années 1980. Cette évolution terminologique fonctionne comme un marqueur de modernité (Nay, 1994) impliquant une transmission, un aller-retour d'un message. Plus largement, la diffusion sociétale du vocable communication (Neveu, 2006) implique que sa définition précise perde de son efficacité explicative, son sens se diluant en proportion de sa généralisation dans toutes les activités sociales.

Bien que le concept soit flou (Breton, Proulx, 2002) et renvoie à de multiples activités (transports, médias, télécommunications, informatique, publicité, relations publiques et presse, communication des organisations, internet...), il est heuristiquement opératoire avec le qualificatif public. C'est un échange intermittent entre une logique unilatérale hiérarchique ${ }^{2}$ institutionnellement dominante et un contact des usagers avec l'administration. Ainsi, la communication publique est très majoritairement à sens unique, avec d'un côté des gouvernants qui disposent en permanence de moyens d'action communicationnels institutionnels et d'un autre côté des gouvernés qui n'expriment leur volonté que sporadiquement (élections, consultations).

Aujourd'hui généralisée, la pérennisation de la communication institutionnelle publique fait se poser la question de la définition de cette activité organisationnelle. Ce questionnement posé par des professionnels ${ }^{3}$ et des chercheurs n'intervient qu'ex post. La communication publique naît progressivement en dépit de son flou conceptuel en s'inspirant des modèles d'usage entrepreneuriaux, électoraux (Bessières, 1998). Elle est avant tout une pratique avant

\footnotetext{
${ }^{1}$ Le terme d'information est revenu sur le devant de la scène avec le développement d'internet depuis le milieu des années 1990 en France.

${ }^{2}$ Ceci est lié en partie à la notion de pouvoir politique, public, institutionnel

${ }^{3}$ Plusieurs entretiens de l'auteur critiquent une insuffisance conceptuelle avec des méthodes peu formalisées
} 
d'être un concept théorisé. Aussi, l'exercice de cette communication organisationnelle est difficile à caractériser. Toutefois, elle est souvent définie en fonction de deux qualificatifs : politique et publique. Ils fournissent une assise théorique pour catégoriser des usages et des pratiques. Cette alternative atteste du flottement théorique qui entoure cette communication dont la perception n'est pas totalement stabilisée (champ, finalité).

Cette communication ne bénéficie pas de définitions positives dans le droit et la jurisprudence, en dépit de quelques progrès issus des réformes du code des marchés publics aménageant des modalités plus souples pour la communication. Tout au plus peut-on trouver des interdictions de certaines actions de communication à l'approche des scrutins électoraux en vertu de la loi de 1990. Toutefois, il s'agit de restrictions assez tardives (Bessières, 1998) en comparaison des époques d'émergence de la communication publique ${ }^{4}$ et non d'une définition ${ }^{5}$.

Notre objectif est de mesurer pourquoi le concept de communication publique est devenu aujourd'hui dominant en dépit de ses différentes approches. Notre but est résolument compréhensif et de dépasser les limites ambigües de 1'expression communication publique, de recenser des éléments de théorisation ou de représentation de cette activité. Il s'agit de montrer qu'elle est à la fois un facteur de légitimation pour ses acteurs directs (communicants) mais également pour ses analystes théoriques. Ainsi, la définition de cette communication est à relier à un processus d'institutionnalisation, en tant que support de confortement d'un secteur professionnel et d'un champ universitaire constitutif d'une discipline. Mais c'est également conjointement une composante des institutions publiques et de leur fonctionnement, aujourd'hui renforcée par un intérêt managérial plus prégnant. La méthodologie implique une analyse théorique du concept et de ses cadres au travers de conceptualisations d'auteurs universitaires et professionnels relativement peu nombreux. De ce point de vue, la communication publique constitue un parent pauvre de la recherche en comparaison d'autres formes de communication ${ }^{6}$.

\footnotetext{
${ }^{4}$ Création de services dans les années 1970, amorce de généralisation la décennie suivante

${ }^{5}$ À notre connaissance en Europe, seule l'Italie s'est dotée d'une loi régissant la communication publique qui a eu des conséquences en matière de formation (loi du 7 juin $2000 \mathrm{n}$. 150).

${ }^{6}$ La littérature est plus abondante en communication d'entreprise, marketing ou ressources humaines
} 
Cette communication ressort également de son appartenance à des organisations publiques qui contribuent à la conditionner et à en définir les spécificités. Au demeurant, le succès de l'appellation communication publique repose sur sa capacité à dépasser les frontières académiques établies parce qu'elles ne peuvent se réduire à une seule vision. Bien plus, elle intègre une pluralité d'enjeux et d'applications regroupées sous une même bannière.

La communication publique : dominante dans un champ de lutte de définition

Les définitions de la communication institutionnelle publique constituent un enjeu de lutte disciplinaire. Ces logiques de positionnement correspondent à des modèles d'interprétation d'une réalité sociale en croissance. Mais elles manquent de précision, même si l'appellation communication publique bénéficie du soutien des acteurs sociaux qui la mettent en œuvre?

Une spécification communication publique pour les $S^{8} C^{8}$ et les professionnels.

Certains auteurs cherchent à définir la communication institutionnelle comme une communication publique, en la différenciant d'une communication politique. Ainsi, Bernard Miège (1989) définit la communication publique comme différente de la communication politique bien qu'il admette des chevauchements inévitables dans la recherche d'une adhésion à une politique menée par l'Etat par une campagne de communication: "Il faut d'abord distinguer la communication publique de la communication politique avec laquelle elle est souvent confondue ». "La communication publique est donc multiple, même si les effets qu'elle recherche se complètent plus qu'ils ne s'opposent ou se concurrencent». Pour caractériser cette communication institutionnelle, des d'analystes prônent une séparation théorique entre les deux appellations parce qu'elle entraîne un choix de recrutement entre des professionnels (modèle actuel) d'un côté ou des militants politiques (modèle ancien) d'un autre (Pailliart, 1993). On critique également le risque d'une personnalisation de la communication autour de l'élu attentatoire aux finalités publiques (Zémor, 2008), responsable de son assimilation à la communication politique (Miège, 1989).

\footnotetext{
${ }^{7}$ Voir les ouvrages de professionnels reprenant l'appellation (Mégard D., Hartereau A...)

${ }^{8}$ Sciences de l'information et de la communication
} 
C'est l'association éponyme, créée en 1989, qui permet à cette appellation de supplanter celle de communication sociale antérieure (Ollivier-Yaniv, 2000) et son succès auprès du champ de professionnels et des institutions publiques. Selon son fondateur, Pierre Zémor (2008), «la communication publique est la communication formelle qui tend à l'échange et au partage d'informations d'utilité publique, ainsi qu'au maintien du lien social, et dont la responsabilité incombe à des institutions publiques »... Ses finalités sont par conséquent les mêmes que celles d'organismes publics, à savoir "d'informer, (faire savoir, rendre compte et faire valoir), d'écouter (les attentes, les interrogations et le débat public), de contribuer à assurer la relation sociale (sentiment d'appartenance collectif, prise en considération du citoyen en tant qu'acteur) et d'accompagner les changements tant comportementaux que d'organisation sociale ». Elle s'attache aux organisations publiques. Cette conception est également reprise en Italie et en Belgique ${ }^{9}$ avec un sens plus restreint que dans le contexte nord américain où elle recouvre les institutions publiques, mais aussi plus largement les médias, les entreprises, les mouvements et les groupes qui interviennent sur la place publique (Beauchamp, 1998). La spécificité de la communication publique en France est donc majoritairement organique, c'est le critère juridique du secteur public (son statut) et/ou sa nature non concurrentielle (son comportement).

Ce caractère public fonde sa spécificité en l'écartant de la publicité et de la communication commerciale, trop simplificatrice de la complexité du service public (Zémor, 2008). Incontestablement, la communication publique s'oppose au privé dans un vaste champ organisationnel qui regroupe les communications de toutes les institutions publiques : ministères, entreprises publiques, établissements publics, collectivités territoriales (Bille, 1991). Plus encore, cette communication relève du monopole de l'institution dans son champ de compétences, ce qui change la portée de celle-ci par rapport à la communication d'entreprise ${ }^{10}$ : elle bénéficie d'une emprise beaucoup plus forte. Cependant, elle est généralement moins précise en raison de l'étendue des mesures publiques qui touchent des catégories larges de population et généralement le grand public (Hallé, 1994). Nous avons pu vérifier lors de divers entretiens que cette communication est souvent peu ciblée, en comparaison de la

\footnotetext{
9 Grâce aux associations Comunicazione Pubblica et Communication Publique Wallonie-Bruxelles

${ }^{10}$ Voir les travaux de Christian Le Moënne en particulier
} 
communication commerciale, au motif de l'intérêt général. Tout se passe comme si le principe de droit public d'égalité de traitement était contradictoire avec les cibles précises de catégories socioprofessionnelles. C'est une communication adressée à un ou des grands publics relevant de découpages de compétences administratives générales (Bessières, 2000). Enfin, des contraintes de gestion publique contribuent également à la définir : le droit (Bille, 1991), le code des marchés publics, la concertation avec un représentant politique (élu ou ministre) ${ }^{11}$.

\section{Des revendications d'intégration dans la science politique.}

Nombre d'auteurs politistes témoignent d'une logique de marquage de champ disciplinaire. La communication institutionnelle publique étant regardée comme une politique publique ou une communication politique, catégories préexistantes.

Pour parler de politique publique, à la base il y a toujours l'identification d'un problème sujet à débat appelant une régulation politique. Des maux sociaux deviennent ainsi des problèmes politiques (Muller, 2008). Ce n'est généralement pas le cas en matière de communication : les exemples de négociations participatives sont marginaux et ponctuels entre acteurs de la décision publique ${ }^{12}$. Pour certains auteurs (Le Bart, 1994 ; Dubois 1991), la communication est sensée apporter une réponse à des besoins d'information présumés des usagers en donnant à voir les autres politiques publiques. Un tel besoin n'est pourtant pas démontré, faute de revendications en ce sens exprimées de façon générale par les populations locales ou nationales : la communication publique ne représente pas un problème collectif en soi (Nay, 1994a) de nature à expliquer son institutionnalisation et sa pérennisation. C'est là une restriction fondamentale à la possibilité d'une analyse de la communication locale comme une politique publique. Dans ces conditions, c'est bien plus la logique de l'offre, issue du pouvoir politique qui prime sur celle d'une hypothétique demande de la population. Le besoin de communication organisationnelle ressort plutôt d'un souci de légitimation institutionnelle et politique ou de gestion et de contrôle symbolique de

\footnotetext{
${ }^{11}$ Entretien d'un responsable du salon professionnel Cap' Com.

${ }^{12}$ Cette possibilité existe quelques fois, au niveau de l'État dans les ministères de l'Equipement et de l'Environnement, où certaines communications ont été négociées entre les fonctionnaires et les associations qui avaient décidé auparavant des actions des politiques publiques (Gentès, 1996). Mais, les exemples de corporatisme de la décision de la communication sont rares
} 
Dossier : Repenser la communication des organisations publiques

l'organisation (Bessières, 1998).

On peut concevoir qu'un effet d'annonce soit considéré comme une politique publique qui identifie des actes, mais aussi à la limite leur absence en faisant croire que l'on agit au moyen de supports de communication (Thoenig, 1985). On voit bien qu'une telle possibilité d'analyse de la communication s'adresse à des actions ponctuelles et non pas à une pratique généralisée. Plus largement, la communication n'est pas une politique publique dans les entreprises, ni dans la communication électorale. Aussi, on ne saisit pas bien ce qui fondamentalement impliquerait de dissocier la communication des institutions publiques des autres types de communication d'organisation. En ce sens, elle est seconde, "intersectorielle », "englobante », et "il n'est pas possible de la dissocier des politiques sectorielles» (Nay, 1994ab) qu'elle met en scène, ni d'évaluer facilement son impact spécifique (Dubois, 1991) des actions ou des services qu'elle promeut.

Pour autant, fondamentalement, cette activité organisationnelle publique a toujours une signification politique. Elle est placée sous le contrôle d'un homme politique (ministre, élu) ou d'un dirigeant tirant sa légitimité d'une nomination politique. Elle sera jugée sur son impact dans l'opinion, sur l'image de l'institution, voire sur celle du dirigeant politique placé à sa tête (Laufer, Burlaud, 1980). Cette communication est de facto conditionnée par la notion de mandat, à ce titre elle est politique. Nombre d'auteurs du champ disciplinaire science politique classent d'entrée de jeu cette communication institutionnelle comme une branche de la communication politique dans leurs ouvrages (Currap, 1991 ; Gerstlé, 2008).

Aussi, certains n'hésitent pas à «... définir la communication politique locale comme une série de méthodes et de techniques visant à mobiliser des formes symboliques (des images, des mots, des attitudes...) représentant une réalité suggérée que le pouvoir politique local voudrait imposer comme référent commun", de nature à répondre à la curiosité de la population, pour transmettre des valeurs, culturelles et politiques, tout en étant un élément de distraction (Nay, 1994a). Il en découle que cette communication est plurielle dans ses utilisations - communication des élus, des institutions, des politiques publiques - mais avec également dans ses objectifs d'information (donner le sens d'une action), de promotion (agir sur les perceptions du public), de socialisation (diffusion de valeurs et de modèles de comportement). Ainsi, la communication locale comporte toujours une valorisation implicite des actions des élus décideurs et l'activation 
de l'identité locale (Chevalier, 1991). Les édiles sont présentés selon un discours axé sur des thèmes territoriaux (promotion du développement local, gestion publique..) destinés à mettre en scène une intervention publique locale consensuelle et à minimiser corrélativement les oppositions d'intérêts (Faure, 1994). Cette dimension des pratiques sociales représente la définition de la communication politique au niveau local selon Jean-Baptiste Legavre (1994).

Cependant, fondamentalement, la communication politique est plus vaste que la communication institutionnelle publique. Elle a trait aux relations sociales dans un espace de coopération et de conflit, au lien social en définitive. Elle passe par divers canaux (Gerstlé, 2008) : institutionnels (parlement, administrations...), organisationnels (partis et groupements politiques), médiatiques (presse, écrits, audiovisuels), interpersonnels (groupes sociaux et relations interindividuelles). En effet, " la communication politique est un échange d'information entre les gouvernants et les gouvernés, par des canaux de transmission structurés ou informels » (Cotteret, 2000), afin d'assurer un accord par la mise en commun de valeurs dans le cadre d'un code commun partagé par les deux groupes d'acteurs.

\section{Une communication hybride.}

En dépit des enjeux de définition théorique, la communication se développe dans la pratique des organisations. Il s'agit pour les institutions de se faire connaître (identification) et reconnaître (légitimation), mais aussi de renforcer le consensus électoral. Elle est alors montrée comme publique ou politique. En définitive, ces deux acceptions sont tout aussi recevables, mais toutes deux sont polarisées sur des visions restrictives et trop larges de cette activité. Par exemple, la communication locale appartient à ces deux visions. Elle est incontestablement une communication publique ; l'accent est alors mis sur la dimension institutionnelle des organismes qui l'utilisent. Elle est très efficace pour l'institutionnalisation en la rattachant au vaste champ de la communication organisationnelle. Toutefois, cette première appellation souffre d'imprécision parce qu'elle ne se réfère pas expressément au niveau politique, pourtant essentiel. En effet, du point de vue de ses finalités, elle représente une communication politique au service d'un exécutif, de dirigeants politico administratifs. Les actions administratives étant alors jaugées in fine en fonction d'une image globale du travail des décideurs. La dénommer comme politique permet de pointer l'intérêt des élus décideurs pour l'existence des services de communication, mais contribue à évacuer la dimension 
institutionnelle absente dans cette appellation (Bessières 1998). Cette double vision théoriquement différenciée, se révèle plus fédératrice au regard du champ des pratiques. En effet, l'exécutif local et national incarne « un compromis magique » entre le politique et l'administratif (Zémor, 2008). Aussi, à ce stade, nous définissons la communication institutionnelle publique comme un ensemble de techniques de légitimation constituant une communication publique politique indirecte. Cette définition est en phase avec la réalité organisationnelle.

\section{L'impact organisationnel : vers changement de paradigme ?}

Le travail social de la communication institutionnelle publique intègre la structure administrative qu'elle a pour charge de traduire et qui la conditionne. Elle est récemment plus concernée par une dimension gestionnaire.

\section{Une reconnaissance dans le champ administratif.}

La communication publique est marquée par un souci de management. Elle représente une transformation des relations avec les administrés de l'Etat qui recherche "une certaine qualité de contact... L'Etat est amené à utiliser de nouveaux procédés de gestion, y compris de gestion d'opinion... » (Miège, 1989). Ainsi, plusieurs opérations de communication sont sollicitées pour l'accueil du public, des moyens de présentation et de transmission d'information, des campagnes visant à modifier des comportements (Sécurité routière...). Aussi, le caractère principal de cette communication réside dans son action sur les représentations accompagnant des actions, des applications de règles, des procédures, la prise de décision publique. Elle met en forme des obligations d'information de certaines décisions (publicité des délibérations du conseil municipal), promeut des actions des services délivrés, ou fait connaître l'institution par des campagnes ou actions d'intérêt général (Zémor, 2008): son rôle est modeste. L'image de l'action relève de la communication (Dupoirier, 1994). Aujourd'hui, la communication apparaît reconnue et intégrée par les acteurs comme élément de gestion des services publics. Cette idée est clairement exprimée par un des plus hauts fonctionnaires, le Viceprésident du Conseil d'Etat (Sauvé, 2008), pour peu qu'elle réponde aux motifs d'accroissement du champ des services publics, des évolutions des sociétés contemporaines impliquant une relation à la fois globale (idée de massification) et individuelle (idée de relations personnelles) avec le collectif et les personnes des usagers. On peut souligner également des évolutions des contextes de fonctionnement, avec un approfondissement de la transparence administrative, la 
diffusion de méthodes de gouvernance négociatrices d'origine européenne liées à la communication, le développement de la consultation et de la participation (lois de 1995 et 2002) qui consolident le champ des actions de communication publique dans la gestion institutionnelle.

\section{Une pratique professionnelle bureaucratisée réglementée.}

La communication, élaborée au moyen de services dédiés dans l'organisation publique, est ainsi soumise à des règles de fonctionnement bureaucratisées. Dans la domination bureaucratique décrite par Max Weber, le pouvoir ne relève pas d'un élément bureaucratique mais du "chef $d u$ groupement [qui] occupe une position de détenteur du pouvoir [...] en vertu d'une élection" (Weber, 1971). C'est le cas des institutions publiques. En effet, suivant Max Weber, l'administration bureaucratisée se détermine principalement par la détention et la mise en ouvre de savoirs spécialisés, et notamment par le caractère de permanence (Weber, 1971). Les professionnels de cette communication, correspondent en partie à ce que ce sociologue définissait comme activité de fonctionnaires. Leurs recrutements s'opèrent sur la base d'un contrat et d'une sélection ouverte en fonction de la qualification professionnelle en "constante progression" d'une part, et sur les rémunérations fixes ouvrant droit à la retraite d'autre part. Ces personnels correspondent assez bien à ces caractéristiques. En revanche, ils s'en éloignent car leur proximité des dirigeants politiques les prive d'une liberté personnelle qui ne les soumettrait qu' "aux devoirs objectifs de leur fonction »; ce qui, allié à leur position hiérarchique variable, ne leur ouvre pas mécaniquement une progression de carrière par avancement à l'ancienneté ou selon leur prestation de service. Ils ne sont pas davantage soumis à une discipline stricte et homogène en raison de la diversité de leurs situations statutaire, hiérarchique, financière, ni même à un véritable contrôle formalisé. S'ils ne correspondent pas totalement au modèle d'idéaltype du fonctionnaire formalisé par Max Weber, ils s'en rapprochent.

Le droit public français ${ }^{13}$ participe aujourd'hui de l'intégration organisationnelle de la communication publique qui ne doit plus être personnalisée au moins six mois avant un scrutin national, ni susceptible d'une campagne de promotion publicitaire des réalisations ou de la gestion, ni mettre en place des actions nouvelles. Ces restrictions promeuvent ainsi l'institutionnalisation d'une

${ }^{13}$ Article L-52 du code électoral en particulier 
communication d'apparence publique (Bessières 1998). Ces prescriptions légales, parce qu'elles sont intégrées dans la pratique des organisations (Duran, 1992), sont un puissant facteur de renforcement du groupe des communicateurs publics. Il en résulte que leurs applications concrètes relèvent de l'expertise des professionnels de la communication, rendue plus nécessaire en raison des risques notamment de remise en cause des élections en cas de manquements. Plus largement, l'activité communicationnelle est régie par une norme juridique impersonnelle qui la légitime en définitive (Laufer, Paradeise 1982). L'organisation recourt à des professionnels (Chevalier, 1991) qui connaissent des routines administratives, des conditionnements par l'observation de règles administratives et juridiques. De ce fait, ils contrôlent des "zones d'incertitudes" (Crozier, 1963) par leur savoir-faire, en raison de la centralité transversale de la communication dans les organisations publiques (Bessières, 1998).

\section{Une pratique managériale administrative élargie.}

Récemment, la communication devient une variable managériale, perçue comme un facteur direct de productivité organisationnelle. En effet, le management participatif atteint le secteur des institutions et des administrations publiques. Les mouvements de déréglementation internationale et le développement de la mondialisation des marchés ont contesté l'efficacité de l'économie publique. La résultante de ces évolutions est une contrainte intégrée variablement par les États européens d'alignement sur les modes de gestion du privé et les critères concurrentiels du marché. Cette crise de la gestion publique centralisée s'est traduite à compter des années 1980 par les processus de décentralisation disqualifiant les modes de management bureaucratiques traditionnels fonctionnant sur un usage de masse de services standardisés. En réponse, une logique de différenciation recherchant une qualité du travail et de service comparable au secteur privé se met en place. "De sorte que l'exigence d'un management participatif et communicationnel s'est aussi présentée dans les institutions gérées par l'État, qu'elles soient industrielles, «assistancielle » ou « administratives » (Floris, 1996). Cette mutation se manifeste par des recherches de décloisonnement des organisations publiques, d'autonomie et d'initiative, des contrôles a posteriori, la mise en place de formes participatives dans le travail et de communication institutionnelle. C'est une nouveauté dans la tradition d'unilatéralité des pouvoirs institutionnels fondée sur une organisation pyramidale hiérarchique exprimant les prérogatives de la puissance publique. 
Le secteur public connaît une large diffusion d'une vision entrepreneuriale devenue un nouveau critère de légitimité. Le management figure un langage adopté par les élites au moment de la perte de légitimé économique du secteur public (Laufer, Burlaud, 1980). La légitimation des moyens ne peut plus se fonder sur le statut public, ni sur des contrôles inopérants face à l'ampleur des activités publiques. Ainsi, l'administration est amenée à rechercher un renouvellement de sa légitimité en permanence, en favorisant un consensus des usagers sur les fins et les résultats (Laufer, Paradeise, 1982) au moyen de la communication. Mais peut-on dire que l'on passe d'une communication bureaucratique à une communication managériale? Récemment la nouvelle gestion publique ${ }^{14}$ s'oppose ainsi à la gestion administrative traditionnelle (bureaucratie wébérienne) critiquée pour son manque de souplesse lié au respect de règles impersonnelles. Elle se manifeste par l'introduction d'instruments de gestion du privé opérant une transformation vers une organisation entrepreneuriale tournée vers la performance (output) et non plus sur les ressources (intrant) (Finger, Ruchat, 1997), recherchant efficience et efficacité. Déjà, on note des exemples d'indicateurs de performance propres à la communication. Dans ce contexte, la légitimité même de la communication dans le fonctionnement des organisations publiques est plus fortement questionnée aujourd'hui par la logique de performance portée par la $\operatorname{LOLF}^{15}$ qui pousse les administrations à la modernisation et à la gestion par la performance. Le but principal réside dans la recherche d'un meilleur pilotage des dépenses et d'une responsabilité accrue des gestionnaires. Les budgets sont votés par des programmes basés sur des objectifs mesurables, assortis d'indicateurs de résultat de communication publique. Les premiers indicateurs apparaissent impropres à retracer l'ensemble des activités communicationnelles. On note l'empirie et une grande diversité de critères choisis. Ils sont pour beaucoup auto définis par des acteurs de la communication publique $^{16}$.

$\mathrm{Au}$ total, la variété des définitions témoigne d'une légitimation théorique des pratiques organisationnelles de communication. Ces deux mouvements concourent à l'institutionnalisation de la communication publique au même titre que son intégration dans les

\footnotetext{
${ }^{14}$ New public management

${ }^{15}$ Loi d'organisation de la loi de finance

${ }^{16}$ Ces indicateurs sont modestes aujourd'hui, des expérimentations ont été mises en place par exemple au ministère des Affaires sociales et au SIG
} 
structures administratives. Ce processus de construction d'un champ social se renforce en tant que discipline entendue comme la conjonction des savoirs professionnels et universitaires labellisant un secteur professionnel. L'émergence de diplômes universitaires spécialisés en communication publique poursuit cette dynamique. «La formation, comme le dit Khun, consiste à apprendre le paradigme, à s'enfermer l'esprit à l'intérieur d'une discipline » (Latour, 1994). Cette discipline, encore floue, permet des passages entre les sciences de l'information et de la communication, la science politique, les sciences de gestion par le jeu des qualifications d'une pratique organisationnelle. De ce point de vue, au classique paradigme bureaucratique wébérien s'ajoute celui du nouveau management public. Tout se passe comme si la communication publique, supportée par le champ de ses professionnels et aussi celui de ses analystes, accroît son emprise grâce à sa plasticité adaptative. Elle est en ce sens toujours une marque de modernité depuis une vingtaine d'années.

\section{Bibliographie}

BEAUCHAMP M., Communication publique et société, Canada, Gaëtan Morin, 1998 (1991), 403 p.

BESSIERES D., Un retour du local instrumentalisé, Quaderni, $\mathrm{n}^{\circ} 42$ 2000, pp. 5-16

BESSIERES D., L'institutionnalisation de la communication locale : le cas des échelons décentralisés départementaux, régionaux, parisiens franciliens. Thèse de science politique, Université Paris I Panthéon Sorbonne, 1998, 531 p.

BILLE J., Vers la banalisation des procédures, in La communication publique, RFAP n`58, avril-juin 1991, pp.214-215

BOURE R., Quand les collectivités territoriales entrent en communication. CinémAction, n63, mars 1992, pp. 169-174

BRETON P., PROULX S., L'explosion de la communication, Montréal, La découverte/ boréal, coll. science et société, 2002 (1989), $284 \mathrm{p}$.

COTTERET J.-M., Gouvernants et gouvernés. Paris, PUF, 2000 (1973), $175 \mathrm{p}$.

CHEVALIER J., Synthèse in La communication politique, Paris, PUF, CURAPP, 1991, p. 197-209

CROZIER M., Le phénomène bureaucratique. Paris, Seuil, 1963, $381 \mathrm{p}$. 
La définition de la communication publique...

CURRAP La communication politique, Paris, PUF, CURAPP, 1991, $209 \mathrm{p}$.

DUBOIS F., Une politique publique de communication: l'exemple de la promotion du plan triennal de la Ville d'Amiens, in $\mathrm{La}$ communication politique. Paris, PUF, CURAPP, 1991, p. 23-31.

DUPOIRIER E., L'évaluation du fait régional in Les territoires de la communication publique - champs et mesures, Actes du rendez-vous de Poitiers. Conseil Régional de Poitou-Charentes et Observatoire Interrégional du Politique, France 1994, p. 25-32

DURAN P., Piloter l'action publique, avec ou sans le droit. Revue Politique et Management Public, vol. 11, n 4, décembre, 1992, p. 146

FAURE A., Les élus locaux à l'épreuve de la décentralisation - de nouveaux chantiers pour la médiation politique locale. RFSP vol. 44 $n^{\circ} 3$, juin 1994, p. 462-479.

FINGER M., RUCHAT B., Le New Public Management: Etat, administration et politique, pp 33-56, in Pour une nouvelle approche du management public (réflexions autour de Michel Crozier), sous la direction de Finger M., Ruchat B., Ed. Seli Arslam 1997, 252 p.

FLORIS B., La communication managériale - La modernisation symbolique des entreprises, PUG, 1996, $272 \mathrm{p}$.

GENTES A., La communication publique : de la mise en scène à la stratégie, de la norme à la démocratie. Thèse pour le doctorat de science de l'information et de la communication, Université Stendhal Grenoble III, 1996, 487 p.

GERSTLE J., La communication politique. Armand Colin, 2008 (2004), $255 \mathrm{p}$.

HALLÉ J.-C., Les territoires de la communication: communication d'entreprise et communication politique in Les territoires de la communication publique - champs et mesures, Actes du rendez-vous de Poitiers. Conseil Régional de Poitou-Charentes et Observatoire Interrégional du Politique, France 1994, p. 45-53.

LATOUR B., Le métier de chercheur - regard d'un anthropologue, INRA Éditions, 1995, 103 p.

LAUFER R.., BURLAUD A., Management public Gestion et légitimité. Paris, Dalloz, Coll. Gestion Systèmes et Stratégies, 1980, $353 \mathrm{p}$.

LAUFER R.., PARADEISE C., Le prince bureaucrate. Paris, Flammarion, 1982, $353 \mathrm{p}$. 
Dossier : Repenser la communication des organisations publiques

LE BART C., La légitimation des politiques publiques : l'exemple de la communication externe à Fougères. Revue Politique et Management Public, vol. 12, $\mathrm{n}^{\circ}$ 1, mars 1994, p.151-171

LEGAVRE J.-B., L'horizon local de la communication politique retour sur la définition de l'expertise. Politix n ${ }^{\circ} 28$, dec. 1994, p.151171

MIEGE B., La société conquise par la communication. PUG, 1989, $226 \mathrm{p}$.

MULLER P., Les politiques publiques, Paris, PUF, coll. Que sais-je?, 2008 (1994), $128 \mathrm{p}$.

NAY O., Le chant local - Politique de communication et stratégie de développement local à Montpellier (1982-1993), Bordeaux, Les cahiers du CERVL, Série Rapports de recherche nº ${ }^{\circ}$, mai 1994, 151 p.

NAY O., Les enjeux symboliques du développement local : l'exemple de la politique de communication de Montpellier. Politiques et management public, vol 12 n4, décembre 1994, pp. 51-69.

NEVEU E., Une société de communication ? Paris, Montchrétien, coll. clefs politique, 2006, $160 \mathrm{p}$.

THOENIG J.-C., L'analyse des politiques publiques in GRAWITZ M. et LECAS J. Traité de science politique, vol. IV, Paris, PUF, 1985, p. $1-53$

OLLIVIER-YANIV C., L'État communiquant, PUF, 2000, 323 p.

PAILLIART I., Les territoires de la communication, PUG, 1993, $279 \mathrm{p}$.

SAUVE J.-M., Une exigence démocratique et un impératif de bonne gestion in LEMAIRE M. ET ZEMOR P. La communication publique en pratiques (sous la direction de). La documentation Française, 2008, p. 419-423

WEBER M., Economie et société, Tome Premier, Paris, Plon, 1971, $650 \mathrm{p}$.

ZEMOR P., La communication publique. PUF, coll. Que sais-je?, 2008, $128 \mathrm{p}$. 